\title{
Studies of $\mathrm{CdI}_{2}-\mathrm{Bi}_{3}$ microstructures with optical methods, atomic force microscopy and positron annihilation spectroscopy
}

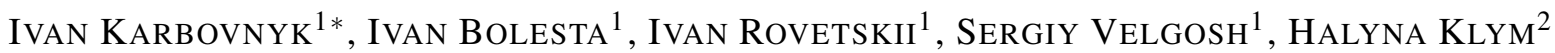 \\ ${ }^{1}$ Ivan Franko National University of Lviv, Ukraine \\ ${ }^{2}$ Lviv Polytechnic National University, Ukraine
}

\begin{abstract}
Results of the comprehensive morphological study of $\mathrm{CdI}_{2}-\mathrm{BiI}_{3}$ layered crystals are presented. Direct AFM observations of micro- and nanostructures formed in the volume of the crystals confirm the predictions made on the basis of positron annihilation spectroscopy studies. The model explaining the possible pores formation mechanism is proposed and validated by the results of luminescence measurements at $8 \mathrm{~K}$.
\end{abstract}

Keywords: layered crystals; microstructures; atomic force microscopy; positron annihilation

(C) Wroclaw University of Technology.

\section{Introduction}

Cadmium iodide crystals are representatives of $\mathrm{MX}_{2}$ halogenides family with pronounced layered structure. This structure is characterized by close hexagonal packing of iodine atoms. Half of the octahedral voids that are available in the structure are filled with cadmium atoms [1]. Strong ioniccovalent bonds between $\mathrm{Cd}$ and I lead to the formation of triple sandwich-like I-Cd-I layers. Due to peculiarities of the structural arrangement of $\mathrm{CdI}_{2}$ there are specific ways of the incorporation of impurities or overstoichiometric $\mathrm{Cd}$ atoms into the crystal lattice. Therefore, a number of reports were devoted to the studies of doped $\mathrm{CdI}_{2}$ crystals and crystals with excess of cadmium (see [24]). In particular, as demonstrated earlier [5], interesting changes in optical absorption are observed when $\mathrm{CdI}_{2}$ is doped with $\mathrm{BiI}_{3}$ (which is another layered compound). Present work is devoted to the investigation of structural and morphological modifications that occur after adding $\mathrm{BiI}_{3}$ into $\mathrm{CdI}_{2}$ crystals; correspondence between these modifica-

*E-mail: ivan_karbovnyck@yahoo.com tion and light emission/absorption processes is also discussed.

To provide further insight into the complex microstructure of layered compound under study, new characterization techniques can be used in complement to traditional ones. One of such novel tools is the positron annihilation lifetime spectroscopy (PALS), a method that can be applied for studying defect structure of surface layers [6]. Analysis of the positron lifetimes (the main goal of PALS method) helps a lot in understanding the electronic processes in the material under study. Combining well-established optical spectroscopy approaches with relatively novel techniques, such as AFM phase analysis and PALS, we expect to shed light on the formation of microstructures within surface layers of cadmium iodide crystals doped with $\mathrm{BiI}_{3}$.

\section{Experimental}

Van der Waals surface morphology of $\mathrm{CdI}_{2}-\mathrm{BiI}_{3}$ crystals was examined exploiting Solver P47-PRO atomic force microscope (AFM) [7], successfully used earlier for the study of nanoclusters in other layered compounds $[8,9]$. With the 
AFM instrument it was possible to achieve $10 \mathrm{~nm}$ in-plane and $0.1 \mathrm{~nm}$ height (z-direction) resolution.

PALS experiments were carried out with ORTEC spectrometer [10]. ${ }^{22} \mathrm{Na}$ isotope was used as a positron source. Measurements were performed at room temperature $(293 \mathrm{~K})$ and ambient humidity (35\%). A series of a few independent experiments were connected with samples of the same prehistory to exclude data scatter because of differences between actual status of samples and uncontrolled instabilities in the experimental setting of PALS spectrometer [11]. The obtained results agreed well with each other within experimental uncertainties, being no more than $\pm 0.005 \mathrm{~ns}$ in lifetimes and \pm 0.01 in component intensities. Obtained positron lifetime spectra were fitted in the frame of two-component positron trapping model. PALS spectra of the investigated layered compound were processed with a standard LT 9.0 computer program [12]. The average positron lifetime $\tau_{a v}$ was determined as a centre of mass of a whole lifetime spectrum according to the well-known expression:

$$
\tau_{a v}=\sum_{i} I_{i} \tau_{i}
$$

where $\tau_{i}$ and $I_{i}$ denote lifetime and intensity of the corresponding fitting components, respectively.

All experimental PALS spectra were fitted by two components with $\tau_{1}$ and $\tau_{2}$ lifetimes and $I_{1}$ and $I_{2}$ intensities, respectively. Then, the positron lifetime in "defect-free" bulk $\tau_{b}$, and positron trapping rate in "defects" $\kappa_{d}$, were calculated using a formalism of two-state positron trapping model $\left(I_{1}+I_{2}=1\right)[6,10]$ :

$$
\begin{gathered}
\tau_{b}=\frac{I_{1}+I_{2}}{\frac{I_{1}}{\tau_{1}}+\frac{I_{2}}{\tau_{2}}} \\
\kappa_{d}=\frac{I_{2}}{I_{1}}\left(\frac{1}{\tau_{b}}-\frac{1}{\tau_{2}}\right)
\end{gathered}
$$

In addition, $\left(\tau_{2}-\tau_{b}\right)$ difference was accepted as a size measure for extended free-volume entities, where positrons are trapped and $\tau_{2} / \tau_{b}$ ratio determined by the nature of these defects was estimated [6].
Photoluminescent properties of $\mathrm{BiI}_{3}$ doped $\mathrm{CdI}_{2}$ crystals were measured with SUPERLUMI HASYLAB facility (Hamburg) using experimental equipment described by Zimmerer [13]. Luminescence was excited by synchrotron radiation extracted from DORIS III accelerator ring. Synchrotron radiation intensity was at the level of $10^{12}$ photons per second. Emission from the sample was focused onto the entrance slit of the BM50 monochromator, set to work in 200 to $1000 \mathrm{~nm}$ spectral range. SI-440-UV photomultiplier in photon counting mode was used as a detector.

\section{Results and discussion}

Fig. 1 shows AFM scans performed on van der Waals surface of $\mathrm{CdI}_{2}-\mathrm{BiI}_{3}$ crystal grown by vapor deposition technique and freshly cleaved surface of $\mathrm{CdI}_{2}-\mathrm{BiI}_{3}$ crystal grown from melt using Bridgman method. The surface of the crystal grown by vapor deposition is covered with hexagonal pores, the shape of which has been determined by hexagonal close packing of the layered structure. Depending on size, the pores can be labeled as "micro" (marked with A letter in Fig. 1) and "nano" (marked with B letter in Fig. 1). Rough estimation provides that an average "nano" pore depth is less that $1 \mathrm{~nm}$, while its average diameter is about $300 \mathrm{~nm}$. As far as "micro" pores are considered, these values are $\sim 10 \mathrm{~nm}$ and $\sim 2 \mu \mathrm{m}$, respectively.

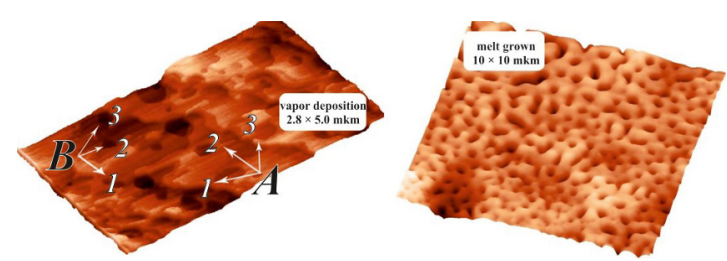

Fig. 1. AFM images showing van der Waals surfaces of $\mathrm{CdI}_{2}-\mathrm{BiI}_{3}$ crystals grown by vapor deposition (left) and crystal grown from melt (right).

Porous structure was observed also while examining the fresh cut of the crystal grown from melt (the right side of Fig. 1). Assuming the preferable shape of pores as oval, pores sizes range from as small as $25 \mathrm{~nm}$ up to as large as $600 \mathrm{~nm}$. The average depth of the pores is around $40 \mathrm{~nm}$. 
Summarizing results obtained using AFM technique one can conclude that micro- and nanosized pores are formed in $\mathrm{CdI}_{2}-\mathrm{BiI}_{3}$ layered compound independently of the crystal growth technique.

In order to clarify the pore formation mechanism, the PALS method described in the previous section was used. Assuming two-state positron trapping model [6], the two components in the fit of the experimental PALS spectra $\left(\tau_{1}, I_{1}\right)$ and $\left(\tau_{2}, I_{2}\right)$ can be associated with the properties of the dominating phase of the material and positron lifetime in the free-volume entities, respectively. In this case, the positron trapping modes (bulk lifetime $\tau_{b}$ and positron trapping rate in defects $\kappa_{d}$ ) can be determined according to the expressions 2 and 3 . The open volume entities free of the electron density are treated as "defects", while hypothetical structure without these entities is treated as "defect-free" bulk (represented by $\tau_{b}$ value). Within accepted two-state positron trapping model, we will focus our further analysis on the second $\left(\tau_{2}, I_{2}\right)$ component, where $\tau_{2}$ lifetime is directly related to the size of free-volume entity (trapping center) and the intensity $I_{2}$ is proportional to the number of such "defects" [10].

Results of the comparative (pure $\mathrm{CdI}_{2}$ vs. CdI$\mathrm{BiI}_{3}$ ) PALS analysis are summarized in Table 1. One can notice that $\mathrm{BiI}_{3}$ additives to pure $\mathrm{CdI}_{2}$ cause an increase in $\tau_{2}$ values indicating an increase in the average free-volume size. At the same time, the $I_{2}$ value decreases in these crystals confirming the decrease in the number of this kind of free-volume entities. Thus, we can conclude that doping of $\mathrm{CdI}_{2}$ with $\mathrm{BiI}_{3}$ leads to the increase of the dimensions of free-volume entities with simultaneous decrease of their total amount. At the same time such positron trapping parameters as $\tau_{a v}$. and $\tau_{b}$ are almost unchanged, while positron trapping rate in defects, $\kappa_{d}$ in $\mathrm{CdI}_{2}-\mathrm{BiI}_{3}$ decreases to $0.30 \mathrm{~ns}$ as compared to $\mathrm{CdI}_{2}$ (Table 1). It means that existing free volume voids in pure $\mathrm{CdI}_{2}$ should be converted into a smaller number of larger voids after doping with $\mathrm{BiI}_{3}$. In other words, we can argue that doping $\mathrm{CdI}_{2}$ crystals with $\mathrm{BiI}_{3}$ causes agglomeration of smaller free-volume entities and formation of complex clusters composed of smaller vacancies. Such process should give rise to the increase of $\tau_{2}$ and the decrease of $I_{2}$. However, in both cases the same type of positron trapping centre is formed $\left(\tau_{2} / \tau_{b}\right.$ values are nearly $0.2 \mathrm{~ns})$. The characteristic size of positron traps estimated due to $\left(\tau_{2}-\tau_{b}\right)$ difference is comparable to the size of atomic vacancy $[6,14]$.

Considering experimental AFM observation of pores in $\mathrm{CdI}_{2}-\mathrm{BiI}_{3}$ crystals and conclusions drawn from PALS measurements, the following model explaining pore formation mechanism at the atomic scale can be proposed. When $\mathrm{CdI}_{2}$ is doped with $\mathrm{BiI}_{3}, \mathrm{Bi}^{3+}$ ions substitute $\mathrm{Cd}^{2+}$ ions at regular lattice sites. Since the charge balance has to be maintained, cadmium vacancies (hereafter denoted as $\left.V_{C d}\right)$ appear. $\mathrm{As} \mathrm{BiI}_{3}$ concentration increases, more compensating combinations are possible, e.g. when four $\mathrm{Bi}^{3+}$ ions substitute for six $\mathrm{Cd}^{2+}$ ions, a pair of $V_{C d}$ vacancies is produced. Generalizing, $2 n \mathrm{Bi}^{3+}$ ions substitute for $2 m \mathrm{Cd}^{2+}$ ions creating $(m-n)$ vacancies. If $m$ is large enough, a number of created vacancies can coagulate and a pore is formed.

One should expect that the above described ion substitution would affect the light emission from $\mathrm{CdI}_{2}-\mathrm{BiI}_{3}$ crystals. In order to verify whether the proposed mechanism is somehow manifested in optical properties, we have measured the photoluminescence spectrum of $\mathrm{CdI}_{2}-\mathrm{BiI}_{3}$. The spectrum was recorded at low temperature $(T=8 \mathrm{~K})$ in order to minimize temperature broadening of emission bands and thus achieve better resolution. The result is depicted in Fig. 2.

Three main peaks can be resolved from the spectrum in Fig. 2. The band at $\sim 2.05 \mathrm{eV}$ is commonly attributed to intrinsic luminescence of $\mathrm{CdI}_{2}[15,16]$. A signature of $\mathrm{BiI}_{3}$ phase is a band peaked at $1.26 \mathrm{eV}$. At the same time we can observe a sharp maximum centered at $1.74 \mathrm{eV}$, which is very likely produced by centres composed of two bismuth ions and a vacancy. Possible structural arrangement corresponding to the abovementioned centre is illustrated in Fig. 3.

The model shown in Fig. 3 corresponds to the simplest charge compensation scheme, according to which three $\mathrm{Cd}^{2+}$ ions are replaced with two $\mathrm{Bi}^{3+}$ ions and a single cation vacancy is created. 
Table 1. Results of PALS analysis for $\mathrm{CdI}_{2}$ and $\mathrm{CdI}_{2}$ doped with $\mathrm{BiI}_{3}$.

\begin{tabular}{ccccccccccc}
\hline & \multicolumn{4}{c}{ Fitting parameters } & \multicolumn{5}{c}{ Positron trapping modes } \\
\cline { 2 - 10 } Sample & $\tau_{1}$ & $I_{1}$ & $\tau_{2}$ & $I_{2}$ & $\tau_{a v}$ & $\tau_{b}$ & $\kappa_{d}$ & $\tau_{2}-\tau_{b}$ & $\tau_{2} / \tau_{b}$ \\
& $n s$ & a.u. & ns & a.u. & ns & $n s$ & $\mathrm{~ns}^{-1}$ & ns & \\
\hline \hline $\mathrm{CdI}_{2}$ & $0.248 \pm 0.004$ & $0.75 \pm 0.01$ & $0.451 \pm 0.004$ & $0.25 \pm 0.01$ & 0.30 & 0.28 & 0.45 & 0.15 & 1.0 \\
$\mathrm{CdI}_{2}-\mathrm{BiI}_{3}$ & $0.267 \pm 0.005$ & $0.82 \pm 0.01$ & $0.481 \pm 0.004$ & $0.18 \pm 0.01$ & 0.31 & 0.29 & 0.30 & 0.19 & 1.6 \\
\hline
\end{tabular}

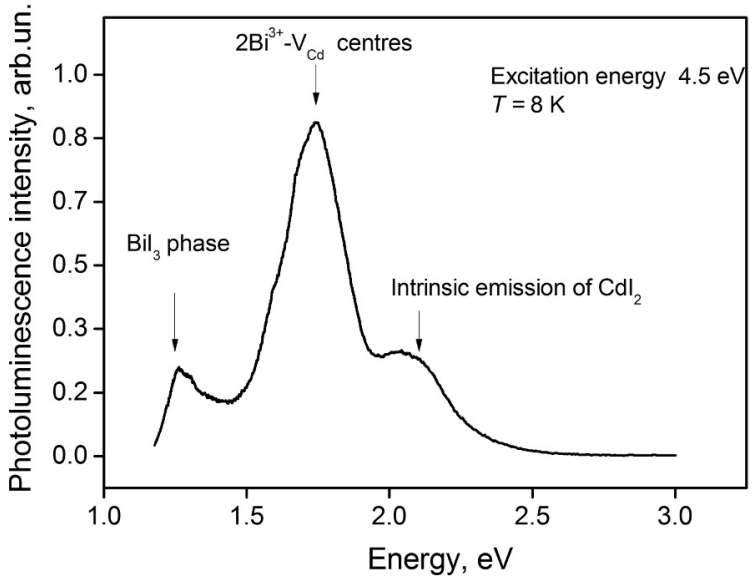

Fig. 2. Low-temperature photoluminescence spectra of $\mathrm{CdI}_{2}-\mathrm{BiI}_{3}$ crystal.

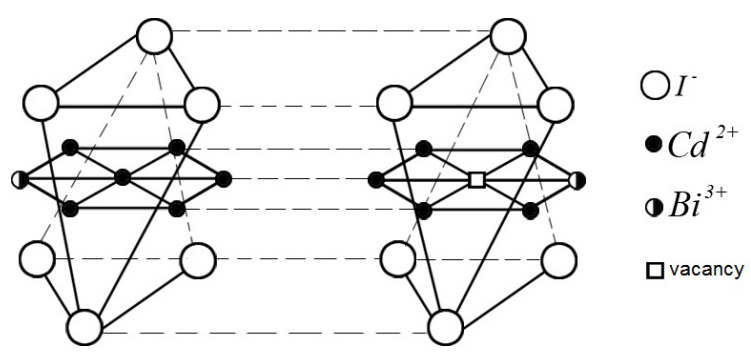

Fig. 3. Model of the luminescence centre formed by ion substitution in $\mathrm{CdI}_{2}-\mathrm{BiI}_{3}$ crystal.

In this case, a cluster composed of two $\mathrm{BiI}_{3}$ molecules and a cadmium vacancy is formed. This model is in a good agreement with our earlier studies, in which it was established that bimolecular clusters might be the source of additional absorption band in $\mathrm{CdI}_{2}-\mathrm{BiI}_{3}$ crystals [5].

\section{Conclusions}

Morphology of van der Waals surface of layered $\mathrm{CdI}_{2}$ crystals doped with $\mathrm{BiI}_{3}$ has been examined using atomic force microscopy. For crystals grown by vapor deposition as well as for those grown from melt, the formation of pores at the submicron scale is observed. Positron annihilation lifetime spectroscopy analysis combined with luminescence measurements speak in favor of the assumption that cadmium vacancies created due to bismuth/cadmium ion substitution are responsible for the pore formation process.

\section{References}

[1] Bozorth R. M., J. Am. Chem. Soc., 44 (1922), 2232.

[2] Chaudhary S.K., Arch. Phys. Res., 3 (2012), 436.

[3] Bellucci S., Bolesta I., Cestelli Guidi M., KarbovnyK I., Lesivciv V., Micciulla F., PASTORE R., Popov A.I., Velgosh S., J. Phys.-Condens. Mat., 19 (2007), 395015.

[4] KRavchuK I.M., Novosad S.S., VoITSEKHOVSKAYA A.M., Inorg. Mater.+, 41 (2005), 779 .

[5] Karbovnyk I., Lesivtsiv V., Bolesta I., Velgosh S., Rovetsky I., Pankratov V., BalasubRamanian C., Popov A.I., Physica B, 413 (2013), 12.

[6] Klym H., Ingram A., Shpotyuk O., Filipecki J., Hadzaman I., Phys. Status Solidi C, 4 (2007), 715.

[7] Binnig G., Quate C.F., Gerber C.H., Phys. Rev. Lett., 56 (1986), 930.

[8] Bellucci S., Bolesta I., KarbovnyK I., Kolych I., Martyniv S., Velgosh S., J. Nanophotonics, 4 (2010), 049503.

[9] Bolesta I.M., Rovetskyi I.N., Partyka M.V., Karbovnyk I.D., Kulyk B.YA., Ukr. J. Phys. Opt., 58 (2013) 490.

[10] Klym H., Ingram A., J. Phys.-Conference Series, 79 (2007), 012014.

[11] Shpotyuk O., Calvez L., Petracovschi E., Klym H., Ingram A., Demchenko P., J. Alloy. Compd., 582 (2014), 323.

[12] KAnSy J., Nucl. Instrum. Meth. A, 374 (1996), 235. 
[13] Zimmerer G., Radiat. Meas., 42 (2007), 859.

[14] Klym H., Ingram A., Hadzaman I., Shpotyuk O., Ceram. Int., 40 (2014), 8561.

[15] Kawabata S., Nakagawa H., J. Lumin., 126 (2007), 48.
[16] Bolesta I.M.,Velgosh S.R., KarbovnYK I.D., Lesivtsiv V.M., Rovetski I.M., Phys. Solid State+, 54 (2012), 2061.

Received 2014-03-27

Accepted 2014-06-06 